$\triangle$ Palabras clave/ Currículo, didáctica. arquitectura, bioclimática.

$\Delta$ Keywords/Curriculum, didactics, architecture, bioclimatics.

$\Delta$ Recepción/ 04 marzo 2016

$\Delta$ Aceptación/ 20 abril 2016

\title{
Formación en bioclimática. Una mirada curricular y didáctica desde el diseño arquitectónico
}

\author{
Training in bioclimatics. A curricular and \\ educational glance from architectural design
}

RES

RESUMEN/ Este artículo trata sobre las estrategias de formación para estudiantes de arquitectura que permitirían incorporar de manera planeada la bioclimática como elemento gramatical del diseño arquitectónico en los cursos de taller de diseño. Entre las conclusiones encontradas, se plantea la necesidad de abordar el problema de la formación específica en bioclimática a través de la propuesta curricular y de didácticas en el aula, fortaleciendo el ejercicio de diseño con la aplicación directa de estudios de factores de comodidad ambiental y eficiencia energética en el proyecto de diseño. En síntesis, se procura concientizar en el proceso de formación sobre la discusión de estas problemáticas, su planteamiento y respuesta a nivel espacial y la evaluación de su impacto. ABSTRACT/ This paper addresses architecture training strategies that would allow for the planned introduction of bioclimatics as a grammatical element of architectural design in design workshop courses. One of the findings is the need to address specific bioclimatics training problems through suggested curriculum and classroom didactics, strengthening the design exercise with the direct application of environmental comfort and energy efficiency factor studies in the design project. In sum, the goal is to trigger awareness during the training process on the discussion of these problems, their spatial approach and answers, and its impact evaluation.

INTRODUCCIÓN. La Arquitectura

Bioclimática se refiere, esencialmente, al vínculo armónico entre el ambiente construido y el clima (Salazar 2011), es decir, a la optimización simultánea de comodidad ambiental y la gestión de recursos naturales a partir de las respuestas arquitectónicas a las variables climáticas. De la definición anterior, se infiere un componente humano y uno medio ambiental.

En relación al componente humano en el diseño, es responsabilidad del arquitecto garantizar las condiciones mínimas de salubridad y comodidad. A nivel internacional han ido surgiendo instituciones y normativas que tienen por objetivo generar lineamientos para favorecer las condiciones mínimas de salubridad y confort en el ambiente construido. Entre ellas destacan las normas ISO 16813 (International Organization for Standardization 2006), ISO 3382-1 (International Organization for Standardization 2009), ISO 18233 (International Organization for Standardization 2006), ISO 16817 (International Organization for Standardization 2012), ISO 7730 (International Organization for Standardization 2005) y ASHRAE Standard 55 (American Society of Heating, Refrigerating and Air-Conditioning Engineers 2010), entre otras.

Con relación al componente ambiental, los gobiernos están aplicando estrategias, 
cada vez con mayor intensidad, para hacer frente a los daños ambientales. En la Conferencia de Naciones Unidas para el Cambio Climático, París-COP21, los líderes mundiales acordaron acciones que se espera que eviten el incremento de la temperatura media de la superficie terrestre por encima de $2^{\circ} \mathrm{C}$ en comparación con los niveles preindustriales, al tiempo que se generen estrategias que posibiliten la adaptación de la humanidad a estos cambios. Desde el diseño arquitectónico se puede contribuir contundentemente a la reducción de gases efecto invernadero, al disminuir la dependencia energética de los edificios, especificar materiales que no impliquen un daño ambiental severo, gestionar el uso racional del agua, prever un manejo adecuado de los residuos, entre otros. Lo anterior evidencia la necesidad de realizar cambios en los procesos de diseño arquitectónico, pues debe asumirse la naturaleza como premisa fundamental. Por lo tanto, debería incorporarse la estética ecológica, que se refiere a la aceptación de soluciones arquitectónicas que implican reflexiones ambientales catalogadas como bellas. Al respecto, Edwards (2008) indica que "[a] pesar de todo, combinando tecnología y ecología es posible proyectar una nueva generación de edificios que produzcan un menor impacto ambiental en todos los frentes". Otros expertos, como Aksamija y Perkins+Will (2013), exponen la importancia del diseño de las fachadas en la obtención del confort ambiental con un uso mínimo de energía y llaman la atención sobre la importancia del conocimiento en variables climáticas.

La Roche (2012), partiendo de la base de que cualquier emisión generada por una edificación es producida por la interacción del edificio y sus componentes con el medio ambiente, propone una metodología de diseño que denomina Carbon-Neutral Design Process (CNDP), que parte de la meta que cada diseñador debe escoger con relación a las emisiones de gases efecto invernadero que generaría su proyecto, la cual debe revisar durante el proceso proyectual.
Otros autores como Guzowski (2010), llaman la atención para que la arquitectura utilice las variables naturales de manera que se retome la relación hombre-naturaleza, se exprese una estética ecológica y se reduzcan a cero las emisiones de gases contaminantes. Pero, adicionalmente, buscan que la arquitectura parta de la premisa de que no debe ser suficiente dejar de hacer aportes negativos, sino que es necesario aportar positivamente.

En ese sentido, las escuelas de arquitectura deben asumir una posición que privilegie, a partir de didácticas en el salón de clase y de estrategias curriculares, la incorporación de

\section{位}

\section{LA BIOCLIMÁTICA EN LOS PROPÓSITOS DE FORMACIÓN.}

La importancia en la inclusión de la bioclimática durante el proceso proyectual radica en el desarrollo de habilidades de pensamiento proyectual relacionadas con la incorporación de elementos formales, técnicos, estéticos, entre otros, a partir de premisas ambientales.

En nuestro contexto, es común que los aspectos bioclimáticos, y en general ambientales, lleguen tarde al diseño, debido a que los procesos de formación en diseño han priorizado otras variables. No obstante, ante la inminente crisis ambiental y ecológica, resulta importante una reflexión sobre la formación en arquitectura: ¿cómo y qué estamos enseñando en el proceso de diseño?

Resulta necesario indagar acerca de la inclusión de la bioclimática en los diseños curriculares a través de asignaturas o cursos de taller de diseño, que evidencien el desarrollo de habilidades de pensamiento bioclimático, es decir, la bioclimática como 'clave gramatical' del ejercicio proyectual. De igual forma, es necesario indagar acerca de las didácticas para la enseñanza de la bioclimática en el taller de diseño.

Entonces, ¿cómo incorporar en los procesos de formación del arquitecto los aspectos clave de la bioclimática, desde el proceso proyectual? ¿Qué tipo de didácticas se requieren para que en el proceso de formación del arquitecto se desarrollen aspectos bioclimáticos del proyecto mismo? ¿En qué etapa del desarrollo proyectual se deben incorporar estos aspectos? Queda claro que es necesario implementar estrategias de enseñanza y aprendizaje que, desde el diseño, lleven a la incorporación de conceptos bioclimáticos para la reflexión técnica y formal desde la idea básica, y repensar los programas existentes en relación a las didácticas, la gestión del profesor y el desempeño del estudiante en el aula.

Cartana y Pereira (2007) Ilaman la atención sobre las dificultades que existen en el taller de diseño, relacionadas con el poco uso de modelos matemáticos o guías que deberían apoyar la enseñanza de la comodidad ambiental en estos escenarios. Los autores identificaron dificultades para incorporar la bioclimática en las fases finales del proyecto. Entretanto, Carlo et al. (2011) evidencian dificultades asociadas al tiempo de duración de un semestre académico en la incorporación de asesores en temas relacionados con la bioclimática en el taller de diseño. Por otro lado, a pesar de que Kowaltowski et al. (2007) Ilaman la atención acerca de posibles restricciones al proceso creativo debido a la integración de variables bioclimáticas durante la enseñanza del diseño arquitectónico, consideramos que se debe interpretar la creatividad como la capacidad de resolver, de manera simultánea e integrada, diversas variables a través del ejercicio de diseño, y no minimizarla al resultado formal del mismo. Finalmente, se requiere proponer instrumentos de medición en el taller de diseño, que permitan procesos de verificación a nivel de la idea básica y a nivel de anteproyecto, tal cual lo expresan Cartana y Pereira (2007).

Finalmente, United Nations Environment Programme (2010) plantea la necesidad de potenciar los procesos de formación en lo pedagógico y curricular en las áreas afines al desarrollo urbanístico de las ciudades, lo que reafirma la necesidad de revisar los procesos de enseñanza y aprendizaje en la formación de profesionales para la arquitectura. 
ESTRATEGIAS PARA LA FORMACIÓN EN BIOCLIMÁTICA. En investigación

previa', se estableció una muestra de

21 programas de arquitectura objeto

de estudio, y se aplicó una encuesta

estructurada a los directores de los

programas para conocer la experiencia

curricular y de formación frente a la

bioclimática. Posteriormente, se realizó un

proceso de análisis e interpretación por

contrastación de resultados a través del

hallazgo de coincidencias y contradicciones

en las respuestas encontradas. Dicho análisis permitió establecer algunas categorías,

utilizadas para clasificar las formas de

actuación curricular de los programas académicos frente a la incorporación de la bioclimática en el proceso de diseño.

Entre los hallazgos mencionados, y en relación a la importancia que se otorga a este tema a nivel del diseño y la gestión curricular, se incluye que el $\mathbf{9 5 . 2 4 \%}$ de las universidades lo considera como importante en el diseño curricular de los programas de Arquitectura. Por otro lado, y en cuanto a los mecanismos utilizados en los programas académicos para la incorporación de la bioclimática, pregunta que quedó abierta para que los Directores de los Programas encuestados mencionaran sus experiencias,

se encontraron 4 formas de incorporación:

\section{Cursos obligatorios en el plan} de estudios: Cursos que el estudiante toma de manera obligatoria para optar al cumplimiento de todos los requisitos del programa. Permiten que el estudiante forme competencias en bioclimática que puedan ser aplicadas de manera indistinta en el taller de diseño. La condición de este tipo de cursos es que el estudiante debe ver los contenidos de forma aislada y luego incorporarlos al taller de diseño y/o a la práctica exigida. Este tipo de curso puede ser teórico o teórico práctico. Al respecto, se encontró que el $9.5 \%$ de los planes de estudio abordados contempla esta alternativa (figura 1).

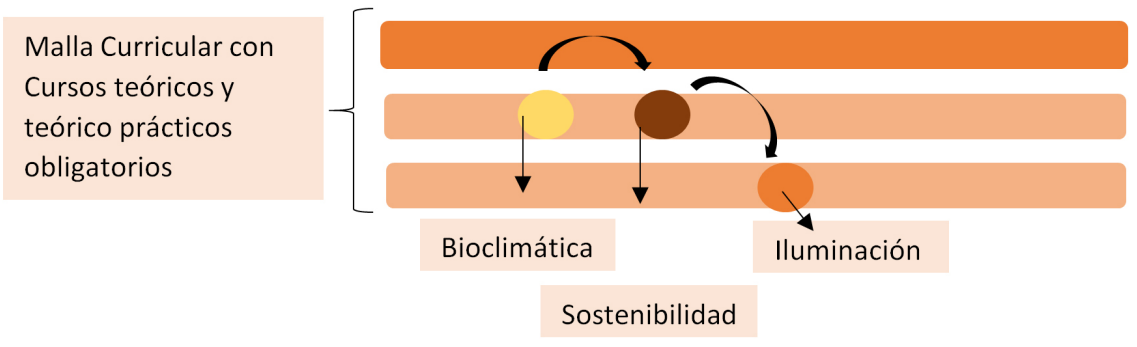

Figura 1. Cursos Obligatorios teórico o teórico práctico (fuente: Los autores).

\section{Cursos}

interrelacionados al

taller del Plan de

estudios o

extracurriculares

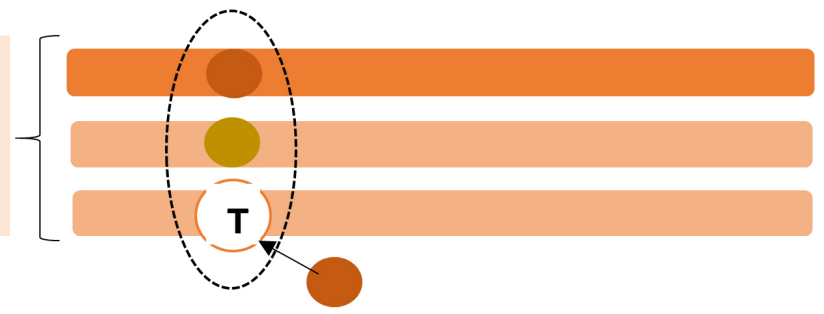

Figura 2. Cursos Obligatorios teórico o teórico práctico (fuente: Los autores).

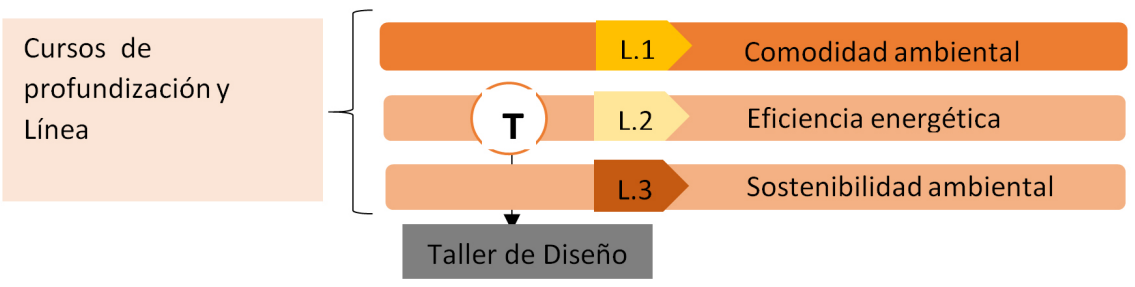

Figura 3. Cursos de profundización y línea (fuente: Los autores).

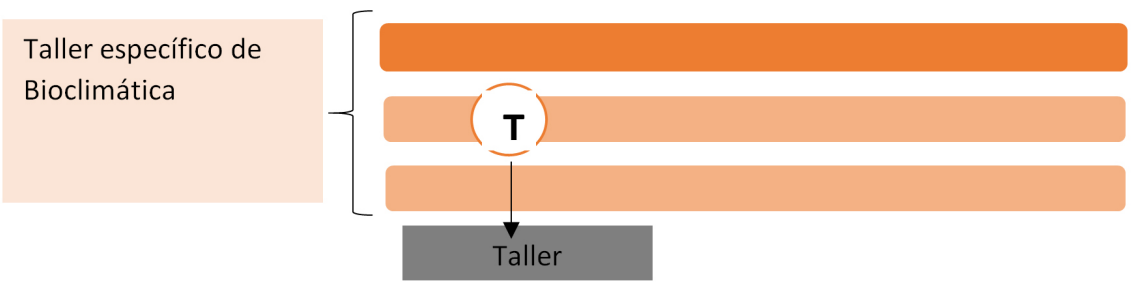

Figura 4. Ialler de bıclimatica (tuente: Los autores). 
2. Cursos y talleres: Ambos espacios de formación u otros de tipo extracurricular. En el $9.5 \%$ de los programas analizados, se incluyen todas las alternativas anteriores en el currículo para la formación de la bioclimática, es decir, se cuenta con cursos electivos, con talleres y se realizan cursos

con prerrequisitos (figura 2).

\section{Cursos electivos: Son tomados por}

los estudiantes para completar procesos de formación de la profesión. Estos cursos son elegidos entre un catálogo abierto. Según el estudio realizado, pueden tener contenido en bioclimática, y se encuentra en el $23 \%$ de los programas de arquitectura analizados (figura 3).

\section{Cursos de taller de diseño en bioclimática:}

de la bioclimática en el taller de diseño existen dos criterios. El primero se refiere a procesos de formación que desarrollan competencias en el estudiante orientadas a la bioclimática solo desde el análisis del lugar, y conllevan a que se realice un estudio del lugar que dé pistas sobre las necesidades de implantación del edificio; análisis desarrollado en todos los ejercicios de diseño, sin hablar de este aspecto como un fenómeno especial del diseño para el edificio (figura 4). Por este rumbo se encuentran el 38\% de los programas analizados. El segundo criterio es el diseño de un taller vertical específico en bioclimática. En el proceso de indagación, esta alternativa de formación solo corresponde al $4.76 \%$ de las universidades encuestadas. Vale la pena decir que esta alternativa se expresó además como optativa, es decir, elegida por los estudiantes entre muchas de las alternativas propuestas para profundización o línea de énfasis a nivel de los talleres de diseño. Ahora bien, cabe la pregunta: ¿Cuál es la mejor manera de formar competencias en el área de la bioclimática? ¿Cómo responder a las exigencias de una realidad que evidencia con urgencia la inclusión en el diseño de edificaciones de variables de confort y calidad de vida de los usuarios?

\section{LA FORMACIÓN EN BIOCLIMÁTICA EN LOS PROGRAMAS DE ARQUITECTURA}

Según las necesidades encontradas en nuestro contexto, se infiere que es preciso incorporar la bioclimática como componente en el currículo. Ello implica la planeación de estrategias que permitan el desarrollo del pensamiento estéticoecológico del estudiante en la gestión curricular. Así, consideramos que los planes de estudio deberían disponer de diferentes escenarios: cursos y talleres con requisitos y créditos obligatorios para el estudio de la bioclimática desde cada una de las asignaturas.

La gestión curricular planeada implica revisar las didácticas y estrategias de enseñanza en el aula y en todos los espacios académicos del programa, especialmente las didácticas usadas en el taller de diseño. Es fundamental la comprensión de la didáctica situada además en el contexto universitario, desde la perspectiva de la didáctica universitaria o didáctica especial: "La didáctica comprometida con lo significativo de los aprendizajes del futuro profesional, con su desarrollo personal y con el potencial de su inteligencia en función de las exigencias del contexto, socio-político" (Díaz 1999). La didáctica debe pensarse orientada a la formación de los saberes profesionales, saberes explícitos y conocimientos tácitos, al saber qué y cómo, al aprender producto del estudio y a aquel aprendido del trabajo, definición que se ha desarrollado desde la perspectiva de Camilloni (2007), para que se puedan identificar las necesidades puntuales de formación del profesional universitario en arquitectura.

A partir de esa mirada, proponemos las siguientes estrategias:

- Formulación de preguntas orientadoras desde la comodidad ambiental.

- Establecimiento de códigos de lenguaje arquitectónico para la representación de la idea desde las condiciones ambientales. Estos códigos en la representación gráfica del proyecto, son estudios relacionados con: usuario, norma, urbanismo, historia, entre otros.

- Construcción de diálogos para la discusión sobre la transformación de los hábitos en relación con la calidad y confort ambiental.

- Verificación de la estética ecológica incorporada en el proceso de diseño desde los mecanismos de evaluación, lo que conlleva a definir indicadores puntuales en el resultado.

- Incorporación de aspectos tecnológicos que evidencien la materialización de la estética planteada.

Así, a partir de la perspectiva de la didáctica especializada, se busca la planificación de la formación desde la intención, los procesos y los recursos -estrategias de calidad de resultados- (Camilloni 2007), incorporando la bioclimática como un aspecto de valor en todo el proceso de diseño. La didáctica desde el taller de diseño, además, no es una suma de actividades y prácticas aisladas, sino más bien la planificación de un proceso completo que busca la transferencia de un conocimiento especializado, técnico y profesional. Estos aspectos a nivel microcurricular e incorporados desde la planeación, se traducen en:

- Explicitar en el propósito de formación del curso el desarrollo de habilidades de diseño situadas en el contexto de la bioclimática, lo que corresponde a la intencionalidad.

- Diseño de contenidos planificados que permitan la conducción de un proceso de diseño desde la idea básica hasta el proyecto (planeación, seguimiento, evaluación y realimentación).

- Definición de un componente 'gramatical nuevo: la estética ecológica.

- Desarrollo de sensibilidad estética y ética frente a la bioclimática.

- Desarrollo de estrategias para la formación de la disciplina desde la bioclimática, como aprender haciendo, aprender a aprender y aprender en colectivo 


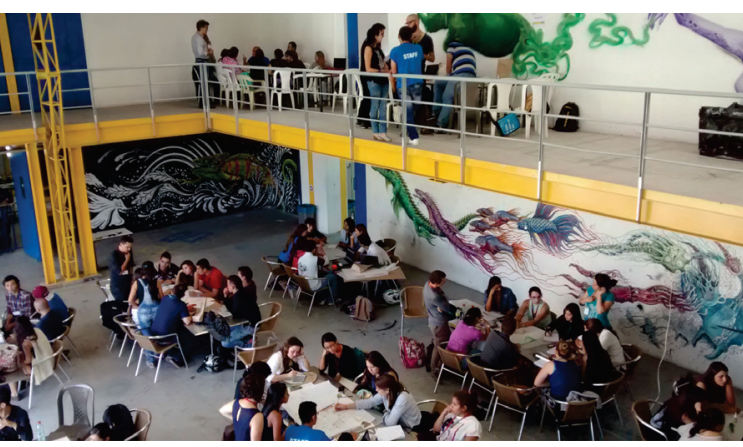

Imagen 1. Taller "La bioclimática como premisa proyectual" llevado a cabo en la Universidad de San Buenaventura, Medellín Colombia (fuente: Los autores).
PILOTO: TALLER-RÁPIDO “LA BIOCLIMÁTICA COMO PREMISA

PROYECTUAL". Como apoyo al proceso

de investigación, se realizó un taller denominado "La Bioclimática como Premisa Proyectual", cuyo objetivo fue abordar el ejercicio arquitectónico priorizando las condicionantes climáticas locales y las variables relacionadas con comodidad ambiental y eficiencia energética. El taller tuvo una duración de 10 horas y contó con la participación de 12 grupos, cada uno con aproximadamente nueve estudiantes (imagen 1). A cada grupo se le pidió que concibiera un espacio de $120-150 \mathrm{~m}^{3}$ que en el exterior tuviera dos árboles de mango adultos y que escogiera, al azar, una variable de cada uno de los cinco rangos de variables (tabla 1). Durante las primeras seis horas, los estudiantes recibieron conferencias simultáneas de expertos en asoleamiento, iluminación natural, ergonomía, acústica, desempeño térmico de materiales y ventilación natural. Además, participaron de asesorías de diseño, didáctica que obligó a los participantes de cada grupo a recibir de manera alterna la información, para buscar una buena solución arquitectónica que respondiera a todas las variables y propició discusiones académicas de diseño al interior de cada grupo.

Tanto en las charlas como en las asesorías, se enfatizó el diseño y la interacción más que la forma arquitectónica, es decir, dónde y cuánto sol, viento, luz, ruido, entre otros. La forma y la fachada fueron consideradas como productos de las necesidades bioclimáticas identificadas. Se abordó el diseño haciendo uso de las herramientas propias del nivel de formación de los estudiantes, en respuesta a los desafíos bioclimáticos. Más allá de la calidad gráfica en la presentación final y de la pertinencia arquitectónica del producto final, se encontraron interesantes propuestas de representación para abordar problemas cotidianos de la comodidad ambiental. En los resultados del grupo 1 (figura 6), se encontraron estrategias encaminadas a favorecer la ventilación y la luz natural, asi como la obstrucción de los rayos del sol. En las figuras 5,6 y 7 , se resalta la composición frente a las variables bioclimáticas en la etapa de diseño, donde no se limita el proceso creativo, sino que se enriquece en su morfología.

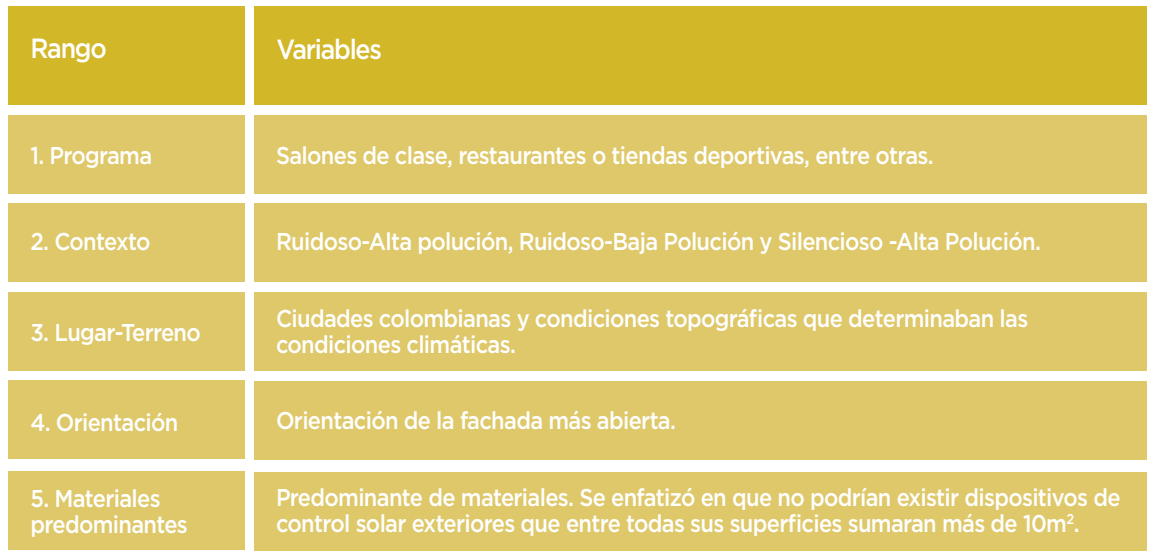

Tabla 1. Variables del Taller "La Bioclimática como Premisa Proyectual" (fuente: Los autores). 


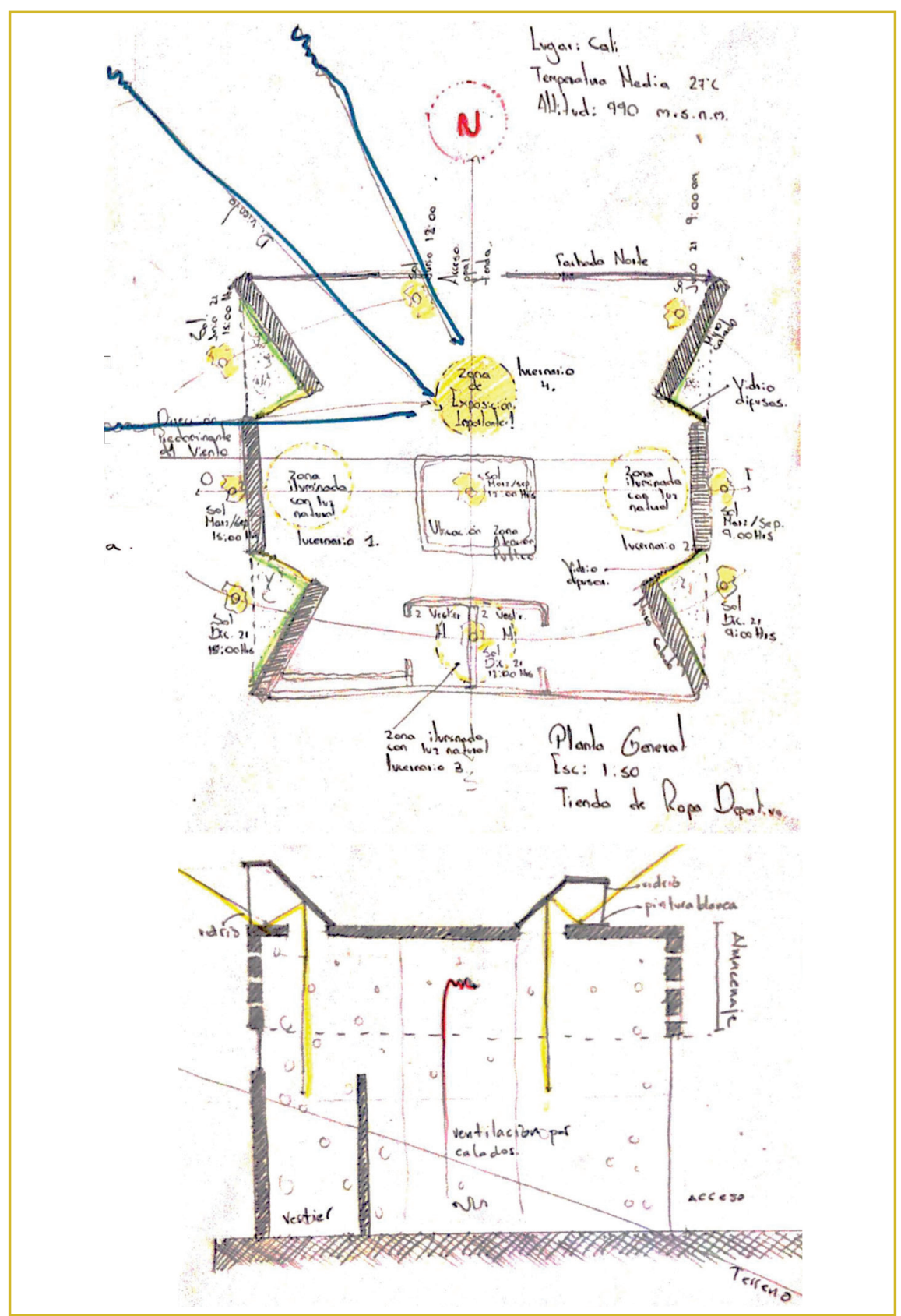

Figura 5. Relación interior-exterior a través de aberturas para iluminar y ventilar. Grupo 1: Bioclimática como premisa proyectual (fuente: Los autores a partir de afiche elaborado por Andrea López, Johan Naranjo, Jessica Atehortúa, Edison Giraldo, Manuela Coronado, Miguel Vélez y Sebastián Vélez).

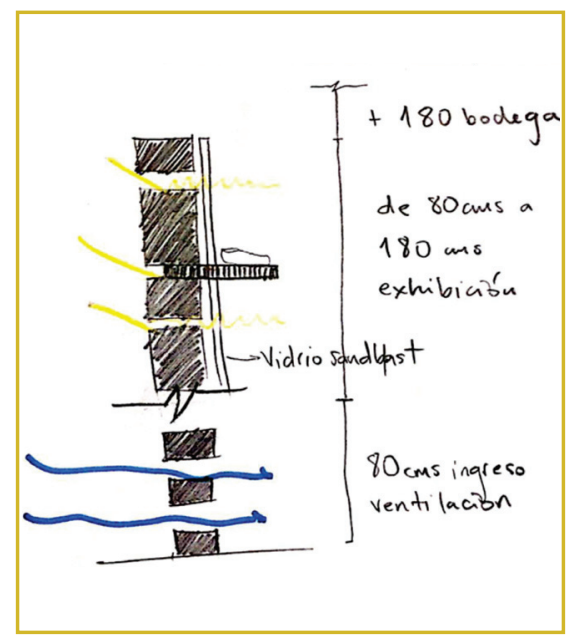

Figura 6. Boceto de diseño de la piel. Grupo 1: Taller La bioclimatica como premisa proyectual (fuente: Los autores a Jessica Atehortúa, Edison Giraldo, Manuela Coronado, Miguel Vélez y Sebastián Vélez).

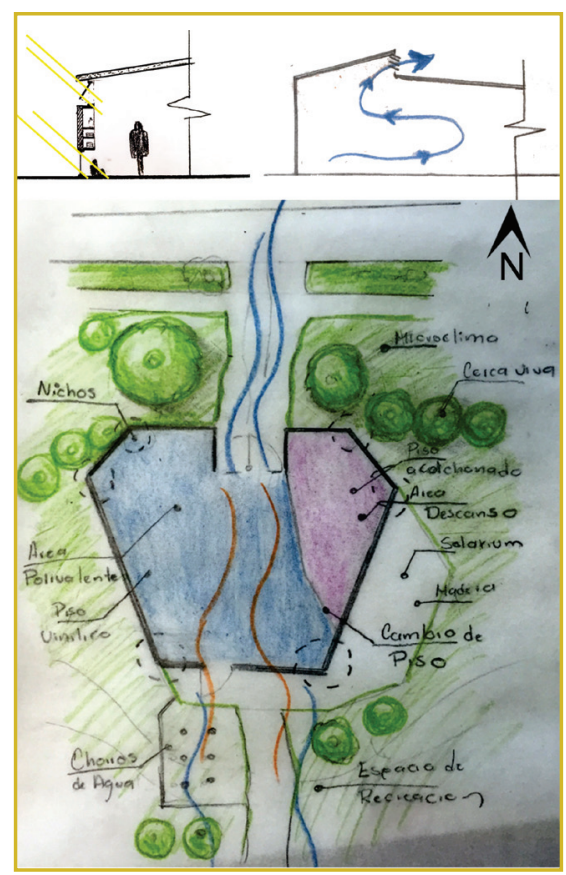

Figura 7. Estrategias de diseño de fachada y de distribución interior en función de la orientación. Grupo 12: Taller de bioclimática como premisa proyectual (fuente: Ana Sánchez, David Restrepo y Daniel Franco). 
CONCLUSIONES. Lo evidenciado en el contexto local apunta a la necesidad de adoptar, desde el taller de diseño de las escuelas de arquitectura, la estética ecológica como un objetivo proyectual. Para ello, dicha estética debe estar visibilizada en el propósito de formación del taller de diseño y debe estar incorporada en las competencias del curso, de tal manera que pueda ser verificable en el diseño. Queda claro, además, que en la formación del arquitecto conviene interrelacionar el taller de diseño con otros cursos que posibiliten un abordaje estético ecológico del estudiante en el proyecto. La bioclimática no deberá ser más un curso electivo, sino un conocimiento que se ofrezca implícitamente en el taller de diseño. Ahora bien, durante el proceso proyectual de los ejercicios típicos de los talleres de diseño, tal como se evidenció en el ejercicio piloto, conviene incorporar la estética ecológica como clave gramatical, que aporta al proyecto reflexiones críticas sobre su relación con el medio ambiente y el mejoramiento de la calidad de vida. Por ejemplo, en todos los proyectos planteados en el ejercicio piloto, se delimitó el problema proyectual a las necesidades bioclimáticas. Esto derivó en que cada búsqueda formal se centrara en la resolución de problemas específicos de asoleamiento, iluminación natural, ventilación natural, aislamiento acústico, etc. De esa manera, la forma terminó siendo el resultado del diseño de la interacción deseada entre usuario y clima. Esto implicó que el estudiante repensara la geometría, los materiales, la orientación, el tamaño de los ambientes, la composición de la fachada, entre otros, en función de las necesidades previamente delimitadas. Se evidenció que incluir la bioclimática como premisa proyectual desde las primeras etapas del proceso de diseño, no necesariamente da como resultado ejercicios rígidos o poco creativos, sino, por el contrario, ejercicios que demuestran diversidad de exploraciones formales para resolver el problema de diseño.

Finalmente, a pesar de que se escapa del alcance del ejercicio piloto, convendría que, en la etapa de anteproyecto, se estimen valores e índices que den cuenta del desempeño térmico, lumínico y acústico del proyecto a través de instrumentos y herramientas de experimentación en el aula, a fin de fortalecer la enseñanza de la bioclimática en la arquitectura.

\section{REFERENCIAS}

Aksamija, A. y Perkins+Will., 2013. Sustainable Facades. Design Methods for High Performance Building Envelopes. Estados Unidos: Wiley

American Society of Heating. Refrigerating and Air-Conditioning Engineers, 2010. ASHRAE Standard 55-2010 Thermal Environmental Conditions for Human Occupancy.

Camilloni, A., 2007. El saber didáctico. Buenos Aires: Editorial Paidós.

Carlo, J., de Araújo, A. y Telles, C. 2011. "Proposta didática para ensino de projeto de arquitetura bioclimática:" Memorias Encac XI. Brasil.

Cartana, R. y Pereira, F., 2007. "Oportunidades e limitaçōes para bioclimatologia aplicada aos projetos arquitetônicos desenvolvidos no meio acadèmico." Memorias. IV Encontro Nacional e // Encontro LatinoAmerica sobre Edificaçỗes e Comunidades Sustentáveis. 277-285.

Diaz, D., 1999. "La didáctica Universitaria. Referencia imprescindible para una enseñanza de calidad." Revista Electrónica Interuniversitaria de Formación del Profesorado. Disponible en: http://aufop.com/aufop/uploaded_ files/articulos/1224326868.pdf (Consultado el 18 de noviembre de 2015).

Edwards, B., 2008. Guía Básica de la Sostenibilidad (segunda edición). Londres: G.G.

Guzowski, M., 2010. Energía Cero: Estética y tecnología con estrategias de dispositivos de ahorro y generación de energía alternativos. Barcelona: Blume.

Organización Internacional de Normalización, 2012. Norma ISO16817:2012 Building environment design -Indoor environment- Design process for visual environment. 2009a. Norma ISO18233:2006. 2009. Acoustics -Application of new measurement methods in building and room acoustics.

2009b. Norma ISO3382-1:2009. 2009. Acoustics -Measurement of room acoustic parametersPart 1: Performance spaces

principles.

2006. Norma ISO16813:2006. Building environment design -Indoor environment- General

2005 Norma ISO 7730:2005 Ergonomics of the thermal environment -Analytical determination

and interpretation of thermal comfort using calculation of the PMV and PPD indices and local thermal comfort criteria.

Kowaltowski, D., Labaki, L., de Paiva, V., Bianchi, G. y Mosch, M., 2007. "Ensino de projeto bioclimático: O papel dos problemas e restrições no processo criativo." Memorias, XI Encac, Brasil. 280-289. La Roche, P., 2010. Carbon neutral architectural design. Estados Unidos: Taylor \& Francis Group. Salazar, J., 2001. Entrevista em 360gradosenconcreto. Disponible en: http://blog.360gradosenconcreto.com/ arquitectura-bioclimatica-y-sostenible-entrevista-con-jorge-hernan-salazar/ (Consultada el 12 de abril de 2016

Programa de las Naciones Unidas para el Medio Ambiente (UNEP), 2010. Guidelines on education policy for sustainable built environments. Disponible en. http://www unep.org/pdf/SBCl Education Policy Guidelines_2010.pdf (Consultada el 19 de noviembre de 2014). 